\title{
Responsible referencing
}

\author{
Careful construction of reference lists is the responsibility of every scientist.
}

Peer reviewers for Nature Methods are vital not only for critiquing experimental designs, checking whether conclusions are supported by results, and gauging the potential impact of work submitted to the journal. Reviewers also often comment on whether new research has been properly put in context with previous publications, and they sometimes identify key papers that authors have overlooked.

An author's response to reviewer comments about failing to cite relevant literature often goes something like, "because of the limitations of Nature Methods formatting, we apologize that we did not initially cite the papers that the reviewer mentions." Yes, we do set limits on the number of references in research papers. This is not intended to be a draconian policy; rather, we do this in part to encourage authors to construct thoughtful, streamlined reference lists where only the most appropriate papers are cited. If authors or reviewers argue strongly that additional references beyond our limits must be cited, we do make exceptions. We also allow further citations in the Online Methods section and of course in supplementary materials.

Authors have a duty to give appropriate credit to previous work. Almost every scientist stands on the shoulders of those who came before, to paraphrase a quote made by Isaac Newton in the 17th century. Previous research is almost always the motivation for the work being reported; therefore, constructing a reference list should not be treated as an afterthought when finalizing a paper for submission. Putting new research in its proper context by conscientious referencing is as much a part of ethical scientific practice as is a clear and honest presentation of the data. Purposeful exclusion of directly relevant references is itself a form of research misconduct.

We hope that this is rarely the case, and that most failures to cite relevant work are unintentional. Here are some suggestions for authors assembling a reference list.

First, keep up with the literature in your field. Scientists who stay on top of the literature are much less likely to miss a key reference, though this is of course more challenging in multidisciplinary research. Subscriptions to journal table of contents alerts, RSS feeds, and recommendation engines such as ReadCube (which partners with Springer Nature) and PubChase that deliver relevant content directly to your inbox can help tame the literature flood. Reading comprehensive review articles and performing extensive PubMed (or Scopus, or Web of Science, etc.) searches will help ensure that you don't miss any older references.

Second, actually read the papers you cite. Papers should not be cited just because they represent an example from a prominent journal, nor should reference lists from published papers simply be copied. These practices lead to artificially inflated citations for certain papers at the expense of more relevant work. Take care to cite the conceptual breakthrough paper(s), not only papers that feature a minor variation or later development (except where that variation is directly relevant, of course).

Third, cite review articles judiciously. When describing a broad topic, it is useful to cite an authoritative review. However, if you are discussing a specific finding or method, it's worth taking the trouble to track down the original reference. It is not only important to give credit to the discoverer, but it also helps readers to rapidly find the appropriate paper rather than sending them on a chase through multiple reference lists, which can be a major annoyance.

Fourth, put citations into appropriate context in the text. Authors should not exaggerate the significance of their own findings, unfairly diminish the findings of others, or worse, use a citation to claim a position that the referenced paper does not in fact support. Citing relevant references without providing proper perspective is not helpful, and it is almost as serious a problem as leaving the reference out completely.

Finally, keep it objective. Reference lists should be balanced and not weighted to citing work predominantly from one's own lab in an attempt to boost personal citation metrics. Further, in cases of scientific competition, disagreements, or personal conflicts, a responsible scientist still discusses work fairly from all sides. Failure to do so can strain relationships between researchers and reflect poorly on a scientist's reputation, in addition to distorting the scientific record.

The process of editorial and peer review is far from perfect; despite our careful attention to presentation in the papers we publish, we and our reviewers do not catch every instance of poor referencing. In this day of tight research funding and where undue emphasis on citation metrics is often applied in hiring and career advancement decisions, responsible referencing should be at the forefront of every scientist's mind. 